\title{
Fast-track multidisciplinary treatment versus conventional treatment for colorectal cancer: a multicenter, open-label randomized controlled study
}

Jun $\mathrm{Li}^{1+}$, Xiang-Xing Kong ${ }^{1 \dagger}$, Jiao-Jiao Zhou ${ }^{1+}$, Yong-Mao Song ${ }^{1}$, Xue-Feng Huang ${ }^{2}$, Gen-Hai Li ${ }^{3}$, Xiao-Jiang Ying ${ }^{4}$, Xiao-Yu Dai ${ }^{5}$, Min Lu ${ }^{6}$, Kai Jiang ${ }^{1}$, Dong-Liang Fu' ${ }^{1}$, Xin-Lin Li', Jin-Jie He ${ }^{1}$, Jian-Wei Wang ${ }^{1}$, Li-Feng Sun ${ }^{1}$, Dong Xu ${ }^{1}$, Jing-Yan Xü, Min Chen², Yu Tian', Jing-Song Li ${ }^{8}$, Min Yan${ }^{7}$, Ying Yuan ${ }^{9}$ and Ke-Feng Ding ${ }^{1 *}$ (D)

\begin{abstract}
Background: Laparoscopic surgery, fast-track perioperative treatment and XELOX chemotherapy are effective strategies for shortening the duration of hospital stay for cancer patients. This trial aimed to clarify the safety and efficacy of the fast-track multidisciplinary treatment (FTMDT) model compared to conventional surgery combined with chemotherapy in Chinese colorectal cancer patients.

Methods: This trial was a prospective randomized controlled study with a $2 \times 2$ balanced factorial design and was conducted at six hospitals. Patients in group 1 (FTMDT) received fast-track perioperative treatment and XELOX adjuvant chemotherapy. Patients in group 2 (conventional treatment) received conventional perioperative treatment and mFOLFOX6 adjuvant chemotherapy. Subgroups $1 \mathrm{a}$ and $2 \mathrm{a}$ had laparoscopic surgery and subgroups $1 \mathrm{~b}$ and $2 \mathrm{~b}$ had open surgery. The primary endpoint was total length of hospital stay during treatment.
\end{abstract}

Results: A total of 374 patients were randomly assigned to the four subgroups, and 342 patients were finally analyzed, including 87 patients in subgroup 1a, 85 in subgroup 1b, 86 in subgroup 2a, and 84 in subgroup 2b. The total hospital stay of group 1 was shorter than that of group 2 [13 days, (IQR, 11-17 days) vs. 23.5 days (IQR, 15-42 days), $P=0.0001]$. Compared to group 2, group 1 had lower surgical costs, fewer in-hospital complications and faster recovery (all $P<$ 0.05). Subgroup 1a showed faster surgical recovery than that of subgroup $1 \mathrm{~b}$ (all $P<0.05$ ). There was no difference in 5-year overall survival between groups 1 and 2 [87.1\% (95\% Cl, 80.7-91.5\%) vs. 87.1\% (95\% Cl, 80.8-91.4\%), $P=0.7420]$.

Conclusions: The FTMDT model, which integrates laparoscopic surgery, fast-track treatment, and XELOX chemotherapy, was the superior model for enhancing the recovery of Chinese patients with colorectal cancer.

Trial registration: ClinicalTrials.gov: NCT01080547, registered on March 4, 2010.

Keywords: Colorectal surgery, Rehabilitation, Colorectal cancer, Randomized controlled trial

\footnotetext{
* Correspondence: dingkefeng@zju.edu.cn

†un Li, Xiang-Xing Kong and Jiao-Jiao Zhou contributed equally to this

work.

${ }^{1}$ Department of Colorectal Surgery and Cancer Institute (Key Laboratory of Cancer Prevention and Intervention, China National Ministry of Education; Key Laboratory of Molecular Biology in Medical Sciences, Zhejiang Province, China), the Second Affiliated Hospital of Zhejiang University School of Medicine, Hangzhou, China

Full list of author information is available at the end of the article
}

(c) The Author(s). 2019 Open Access This article is distributed under the terms of the Creative Commons Attribution 4.0 International License (http://creativecommons.org/licenses/by/4.0/), which permits unrestricted use, distribution, and reproduction in any medium, provided you give appropriate credit to the original author(s) and the source, provide a link to the Creative Commons license, and indicate if changes were made. The Creative Commons Public Domain Dedication waiver (http://creativecommons.org/publicdomain/zero/1.0/) applies to the data made available in this article, unless otherwise stated. 


\section{Background}

Globally, colorectal cancer is the third most common malignancy $[1,2]$. In 2015, there were nearly 376,000 Chinese patients diagnosed with colorectal cancer [3]. Most of these patients could have been cured by radical surgery with or without perioperative chemotherapy and radiotherapy. Fast-track surgery is a combination of several evidence-based perioperative interventions to enhance the recovery of patients after surgery [4]. Studies on fast-track surgery have shown that many conventional perioperative procedures (e.g., bowel preparation and long preoperative fasting) are unnecessary or even harmful to colorectal cancer patients $[5,6]$.

Two European clinical trials, "EnROL" and "LAFA", have shown that fast-track surgery is safe and effective for colorectal cancer patients $[7,8]$. In both trials, fasttrack laparoscopic surgery was the best choice in terms of postoperative hospital stay. Furthermore, four Chinese prospective studies have reported that fast-track surgery effectively accelerates early recovery and reduces the postoperative hospital stay for colorectal cancer patients [9-12]. However, three of the trials have small study populations. Moreover, none of the four studies reported the perioperative procedural details. At present, all reported fast-track surgery studies for colorectal cancer have detailed only the postoperative period (usually only 1 week). However, two-thirds of the patients required 6 months of postoperative adjuvant chemotherapy. Additionally, some procedures in the LAFA and EnROL trials were considered by Chinese surgeons to be radical and were difficult to comply with.

Therefore, we proposed the fast-track multidisciplinary treatment (FTMDT) model in 2010 [13]. This model modifies the fast-track surgical protocols, which are conservative and easy for Chinese surgeons and patients to comply with. Moreover, FTMDT includes fast-track surgery and subsequent adjuvant chemotherapy with capecitabine and oxaliplatin (XELOX). FTMDT can enhance the whole rehabilitation process for patients with colorectal cancer compared to conventional treatment consisting of conventional surgery followed by adjuvant chemotherapy with leucovorin, fluorouracil, and oxaliplatin (FOLFOX). The FTMDT model, which includes more conservative surgical procedures than those in Western countries and covers the overall treatment process, is novel and has never been prospectively compared with conventional treatment. Therefore, this randomized trial aims to compare the safety and efficacy of the FTMDT model versus the conventional model for Chinese patients with colorectal cancer. Moreover, this trial aimed to investigate the total length of hospital stay for patients who received laparoscopic fast-track surgery compared to those who underwent open fast-track surgery.

\section{Methods}

\section{Patients, study design, and randomization}

This was an open-label, prospective randomized controlled study with a $2 \times 2$ balanced factorial design (Clinicaltrials. gov NCT01080547). Eligible patients were randomized (1:1: 1:1) to receive either laparoscopic fast-track surgery followed by XELOX (group 1a), open fast-track surgery followed by XELOX (group 1b), laparoscopic conventional surgery followed by FOLFOX (group 2a), or open conventional surgery followed by FOLFOX (group 2b). Prof. JS L and Dr. Y T, College of Biomedical Engineering and Instrument Science Zhejiang University, oversaw distribution of patients into four study subgroups (1:1:1:1) by simple randomization according with the random number table without stratification. Each participating study center screened and recruited patients. The baseline information was reported to Prof. JS L and Dr. Y T. They performed the patient randomization and informed every center of the randomization results. This trial was approved by the Ethics Committee of Second Affiliated Hospital Zhejiang University School of Medicine (2010LSY No. 6).

The inclusion criteria were patients $\geq 18$ years old with pathologically confirmed colon or upper rectal (distance between the tumor lower margin and anus $>12 \mathrm{~cm}$ ) cancer. All patients were also screened by the investigators and signed informed consents. The exclusion criteria were patients with tumors that could be removed by endoscopic mucosal resection or patients who had a history of malignancy, bowel obstruction, intestinal perforation, evidence of metastasis through physical examination and/or radiological examination, acute disease, acute attack of chronic disease, psychiatric history, spinal deformity that was contraindicated for epidural anesthesia, an American Society of Anesthesiologists (ASA) score IV or higher, or mid-low rectal cancer, or patients who were pregnant.

\section{Study endpoints}

The primary endpoint was the total duration of hospital stay from the time of randomization to 30 days after the last cycle of postoperative chemotherapy. Therefore, it included the days of hospital stay for surgery, adjuvant chemotherapy, and readmission. The postoperative discharge criteria were (1) good pain control (numeric rating scale $\leq 3$ ), (2) tolerance of solid food, and (3) recovery of independent activities of daily living to the patient's preoperative level.

Secondary endpoints included (1) quality of life assessed before surgery and at 1 week, 3 months, and 6 months after surgery via European Organization for Research and Treatment (EORTC) QLQ-C30 and QLQ-CR38 questionnaires; (2) the number of patients with chemotherapy-related adverse events according to the National Cancer Institute Common Terminology Criteria for Adverse Events (NCI CTCAE Version 3.0), which was measured up to 30 days 
after the last administration of chemotherapy; (3) the number of patients with intraoperative and postoperative (measured up to 30 days postoperative) surgical complications, e.g., infection of the incision site, anastomotic leakage, and readmission; and (4) the medical costs (RMB), associated with the whole hospitalization measured up to 30 days after the last surgical procedure or chemotherapy treatment.

Some secondary endpoints that were not prespecified in the study protocol were also analyzed. The surgery duration was calculated as the time from the initial skin incision to the closing of the abdomen. Blood loos was calculated as the blood lost from the time of initial skin incision to the closing of the abdomen. Ambulation onset was recorded as the first time that patient got out of bed postoperatively. Some additional recovery characteristics included the times to first flatus, to defecation, and to resume fluid diet and the duration of the postoperative hospital stay. Thirteen perioperative characteristics, including psychological optimism, anesthesia information, laparoscopy-guided examination, bowel preparation, fasting and oral intake, epidural anesthesia, warming, abdominal drains, fluid infusion, diet, intravenous fluid infusion, nasogastric tube, urethral catheter, and ambulation were assessed to evaluate treatment compliance. Patients who violated more than 10 checkpoints were considered to have not received the allocated intervention. Disease-free survival (DFS) was calculated as the time from randomization to recurrence or death. Overall survival (OS) was the time to death for any reason. All of the above endpoints were compared between groups 1 and 2 to clarify the superiority of FTMDT model over the conventional treatment model. Additionally, all of the above endpoints were compared between subgroups $1 \mathrm{a}$ and $1 \mathrm{~b}$ to clarify the superiority of laparoscopy over open surgery within the set of fast-track surgery procedures.

The FTMDT trial initially included three participating centers, the including Second Affiliated Hospital Zhejiang University School of Medicine, People's Hospital of Shaoxing, and the Second Affiliated Hospital Wenzhou Medicine College. Three additional centers joined in this trial in 2012 to enhance patient recruitment. The new centers were Sir Run Shaw Hospital of Zhejiang University School of Medicine, Ningbo No. 2 Hospital, and People's Hospital of Yuyao. All surgeons taking part in this trial had performed more than 20 laparoscopic operations for colorectal cancer as suggested by the American Society of Colon and Rectal Surgeons [14]. Paper case report forms (CRFs) were collected by the investigators of every participating centers. The CRFs were then collected by the primary investigator Prof. Ding when the patients finished the whole treatment. The investigators of each participating center took responsibility for updating the follow-up data.

\section{Procedures}

The interventions for each group have been previously described in detail [13]. Briefly, patients in group 1 (FTMDT) were given enhanced recovery procedures and 8 cycles of XELOX for high-risk stage II or stage III colorectal cancer. Patients in group 2 (conventional treatment) were given conventional perioperative care and 12 cycles mFOLFOX6 for high-risk stage II or stage III colorectal cancer. The hospital stay for postoperative chemotherapy was 1 day for XELOX and 3 days for mFOLFOX6.

\section{Sample size}

We estimated that the overall duration of the hospital stay for subgroups $1 \mathrm{a}$ and $1 \mathrm{~b}$ would be 14 and 16 days, respectively. Base on our previous research, the overall duration of the hospital stay of groups $2 \mathrm{a}$ and $2 \mathrm{~b}$ were predicted as 46 and 48 days, respectively [15]. With a standard deviation of 6 days for the mean number of hospitalization days, a total sample size of 218 patients would have a power of $>0.85$ to detect a minimum reduction in hospital stay of 2 days among the four groups, using a 5\% significance level. The patients with high-risk stage II or stage III disease who needed adjuvant chemotherapy accounted for $64 \%$ of the total colorectal cancer patients [16]. Therefore, a total of 340 patients, with 85 in each group, were necessary. Considering a $10 \%$ drop-out rate, we planned to recruit 372 patients for randomization to the four subgroups.

\section{Statistical analysis}

Data were analyzed according to the principle of intention to treat. Normal continuous data were presented as the means \pm standard deviations and compared between groups by analysis of variance (ANOVA, $>2$ groups) or unpaired t-test (2 groups). Non-normal distribution data are presented as the medians and interquartile ranges (IQR) and were compared between groups by the MannWhitney $U$ test or the Kruskal-Wallis test. Categorical data were compared between groups by the $\mathrm{X}^{2}$ test or Fisher's exact test for probability. The reported follow-up results were based on the data collected through February 27, 2019. Kaplan-Meier curves of OS and DFS were compared between groups by using the log-rank test. A two-sided $P$-value of 0.05 or less indicated statistical significance. A median difference of more than 10 points in quality of life scoring represented a clinically significant difference [17]. All analyses were performed using STATA (version 12.0; STATA, College Station, TX, USA).

\section{Results}

\section{Patient population}

From April 2010 through June 2014, 612 patients were screened. A total of 374 patients were randomly assigned 
to the four subgroups. Thirty-two patients refused the assigned intervention and withdrew informed consent before surgery. A total of 342 patients were finally analyzed, including 87 patients in subgroup 1a, 85 in subgroup $1 \mathrm{~b}, 86$ in subgroup $2 \mathrm{a}$, and 84 in subgroup $2 \mathrm{~b}$ (Fig. 1). The baseline patients' demographic and clinical characteristics were balanced between groups 1 and 2 . The maximum tumor diameter in subgroup 1a was lower than that in subgroup $1 \mathrm{~b}(P=0.0084)$. The distribution of $\mathrm{pT}$ stages was unequal between subgroup 1a and 1b $(P=0.0210)$. During surgery, a patient in subgroup 1a was found to have peritoneal metastasis (Table 1).

\section{Hospital stay, compliance, surgical recovery, chemotherapy and costs}

The primary endpoint of total hospital stay was shorter in group 1 than in group 2 (13 days vs. 23.5 days, $P=$ $0.0001)$. The total hospital stay of subgroup 1a was similar to that of subgroup $1 \mathrm{~b}$ (13 days vs. 14 days, $P=$ 0.1951, Table 2).

The median number of surgical checkpoints for which the actual procedures carried out were compliant with the planned procedures was lower in group 1 than in group 2 (9 vs. 12 , out of 13 checkpoints, $P=0.0001$ ). The postoperative hospital stay was shorter for group 1 than for group 2 ( 6 days vs. 9 days, $P=0.0001$ ). There was no difference between subgroups $1 \mathrm{a}$ and $1 \mathrm{~b}$ in postoperative hospital stay ( 6 days vs. 6 days, $P=0.2160$ ). The open operation performed with the fast-track protocol (subgroup 1b) resulted in shorter postoperative hospital stays than did the laparoscopic operation performed with the conventional treatment (subgroup 2a) (6 days vs. 8 days, $P=0.0001$ ). The times to resumption of flatus and first defecation were earlier in group 1 than in group $2(P<0.05)$. The times for subgroup 1a were earlier than those in subgroup $1 \mathrm{~b}(P<$ 0.05). The times to resumption of a fluid diet and to ambulation were shorter in group 1 than in group 2 $(P<0.01$, Table 2).

The morbidity of intraoperative complications was similar between groups 1 and $2(P=1.0000)$. The volume of blood loss was lower in group 1 than in group 2 (100 ml vs. $150 \mathrm{ml}, P=0.0014)$. The volume of blood loss in subgroup 1a was lower than that in subgroup $1 \mathrm{~b}$ $(P=0.0150)$. The morbidity of postoperative complications was lower in group 1 than in group $2(6.4 \%$ vs. $14.7 \%, P=0.0140$ ), and there was no significant difference between subgroups $1 \mathrm{a}$ and $1 \mathrm{~b}$. The readmission rates during the 30 days after surgery were similar for groups 1 and 2 (5.8\% vs. $5.3 \%, P=0,8340)$. The surgical cost in group 1 was lower than that in group $2(29,678$ RMB vs. 33,559 RMB, $P=0.0001)$. The surgical cost for subgroup 1a was greater than that for subgroup $1 \mathrm{~b}(P=$ 0.0001 , Table 2). The open fast-tract surgery (subgroup $1 b)$ generated the lowest surgical costs among all four subgroups in the FTMDT trial.

The percent of patients who received adjuvant chemotherapy was similar between groups 1 and $2(62.5 \%$ vs. $63.2 \%, P=0.9100)$. The morbidity of all grades of adverse events was similar between the two groups (94.0\% vs. $96.8 \%, P=0.4990)$. One patient in subgroup 1a and one in subgroup $1 \mathrm{~b}$ died due to cancer metastasis during adjuvant chemotherapy. The chemotherapy cost was similar between groups 1 and $2(100,999$ RMB vs. 104 , 256 RMB, $P=0.1410$, Table 2).

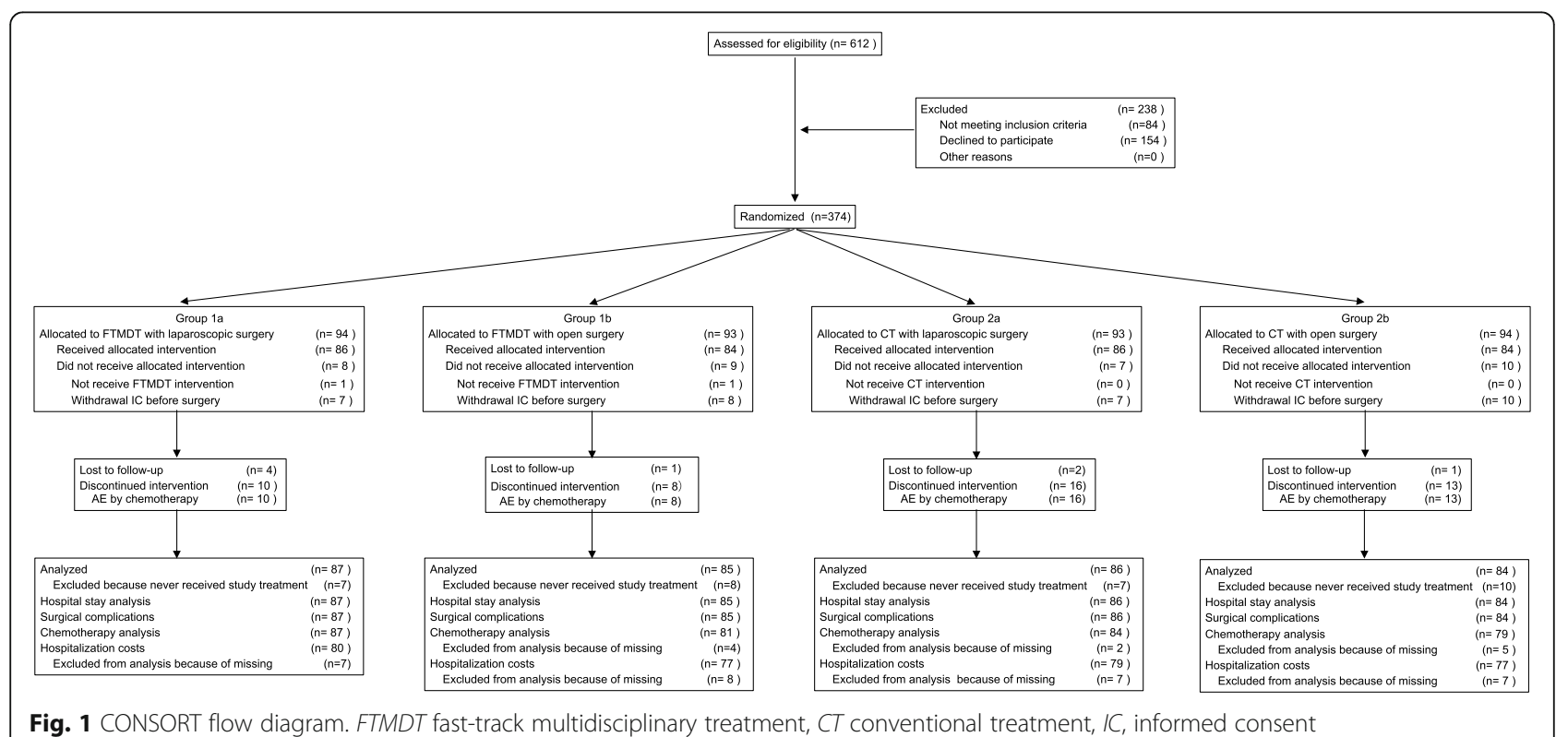

Fig. 1 CONSORT flow diagram. FTMDT fast-track multidisciplinary treatment, CT conventional treatment, IC, informed consent 
Table 1 Baseline Patient Demographic and Clinical Characteristics

\begin{tabular}{|c|c|c|c|c|c|c|}
\hline \multirow[t]{2}{*}{ Characteristic } & \multicolumn{2}{|l|}{ Groups } & \multirow[t]{2}{*}{$P$ value } & \multicolumn{2}{|l|}{ Group1 (FTMDT) } & \multirow[t]{2}{*}{$P$ value } \\
\hline & 1(FTMDT) & 2(Conventional) & & Subgroup 1a & Subgroup $1 \mathrm{~b}$ & \\
\hline Age, years (M, IQR) & $60(53-66.5)$ & $61(54-69)$ & 0.3415 & $61(52-66)$ & $60(54-67)$ & 0.7559 \\
\hline Gender, F/M (F\%) & $62 / 110(36)$ & 67/103 (39) & 0.5210 & $30 / 57(34)$ & $32 / 53(38)$ & 0.6660 \\
\hline $\mathrm{BMI}, \mathrm{M}$ (IQR) & $22.6(20.8-24.6)$ & $22.67(20.6-24.84)$ & 0.9457 & $22.1(21.2-24.8)$ & $23.02(20.65-24.55)$ & 0.7215 \\
\hline ASA grade, n (\%) & & & 0.3920 & & & 0.6340 \\
\hline । & $156(91)$ & $148(87)$ & & $78(90)$ & $78(92)$ & \\
\hline$\|$ & $16(19)$ & $21(12)$ & & $9(10)$ & 7 (8) & \\
\hline III & $0(0)$ & $1(1)$ & & $0(0)$ & $0(0)$ & \\
\hline CEA, ng/mL, M (IQR) & $6.7(3.8-15.2)$ & $5.6(2.9-12.1)$ & 0.3007 & $6.1(3.1-12.9)$ & $7.0(4.8-20.8)$ & 0.2561 \\
\hline CA199, U/mL, M, (IQR) & $28.1(7.0-77.8)$ & $29.8(7.0-51.0)$ & 0.6492 & $16.8(6.1-57.0)$ & $28.3(10.5-103.2)$ & 0.1482 \\
\hline Site of Cancer, n (\%) & & & 0.5110 & & & 0.8810 \\
\hline Ascending colon & $31(18)$ & $35(21)$ & & $18(21)$ & $13(15)$ & \\
\hline Transverse colon & $13(8)$ & $12(7)$ & & $6(7)$ & 7 (8) & \\
\hline Descending colon & $20(12)$ & $24(14)$ & & $11(13)$ & $13(15)$ & \\
\hline Sigmoid colon & $50(29)$ & $38(22)$ & & $24(28)$ & $26(31)$ & \\
\hline Rectum & $54(33)$ & $65(36)$ & & $28(32)$ & $26(31)$ & \\
\hline Maximum tumor diameter, cm, M (IQR) & $4(3.5-5)$ & $4(3-5)$ & 0.3358 & $4(3-5)$ & $5(4-6)$ & 0.0084 \\
\hline Pathologic type, n (\%) & & & & & & 0.1400 \\
\hline Adenocarcinoma & $148(86)$ & $146(86)$ & 0.6260 & $73(84)$ & $75(88)$ & \\
\hline Mucinous adenocarcinoma & $9(5)$ & $13(8)$ & & $6(6.90)$ & $3(4)$ & \\
\hline Other & $3(2)$ & $2(1)$ & & $3(3)$ & $0(0)$ & \\
\hline Missing & $12(7)$ & $9(5)$ & & $5(6)$ & $7(8)$ & \\
\hline Differentiation, n (\%) & & & 0.3840 & & & 0.8480 \\
\hline Well & $20(12)$ & $20(12)$ & & $11(13)$ & $9(11)$ & \\
\hline Moderate & $104(60)$ & $115(68)$ & & $51(59)$ & $53(62)$ & \\
\hline Poor & $34(20)$ & $25(15)$ & & $18(21)$ & $16(19)$ & \\
\hline Missing & $14(8)$ & $10(5)$ & & $7(8)$ & 7 (8) & \\
\hline Lymph nodes, M (IQR) & $15(11-20)$ & $15(12-18)$ & 0.7319 & $14(9-19)$ & $16(11-21)$ & 0.3270 \\
\hline pT stage, n (\%) & & & 0.8070 & & & 0.0210 \\
\hline 1 & $9(5)$ & $9(5)$ & & $7(8)$ & $2(2)$ & \\
\hline 2 & $27(16)$ & $22(13)$ & & $11(13)$ & $16(19)$ & \\
\hline 3 & $91(53)$ & $96(56)$ & & $53(61)$ & $38(45)$ & \\
\hline 4 & $33(19)$ & $28(16)$ & & $11(13)$ & $22(26)$ & \\
\hline Missing & $12(7)$ & $15(10)$ & & $5(6)$ & 7 (8) & \\
\hline pN stage, n (\%) & & & 0.5360 & & & 0.5620 \\
\hline 0 & $97(56)$ & $88(52)$ & & $48(55)$ & $49(58)$ & \\
\hline 1 & $39(23)$ & $46(27)$ & & $23(26)$ & $16(19)$ & \\
\hline 2 & $25(15)$ & $21(12)$ & & $12(14)$ & $13(15)$ & \\
\hline Missing & $11(6)$ & $15(9)$ & & $4(5)$ & 7 (8) & \\
\hline pTNM stage $(n, \%)$ & & & 0.7110 & & & 0.6720 \\
\hline I & $27(16)$ & $23(14)$ & & $13(15)$ & $14(16)$ & \\
\hline$\|$ & $66(38)$ & $63(37)$ & & $32(37)$ & $34(40)$ & \\
\hline
\end{tabular}


Table 1 Baseline Patient Demographic and Clinical Characteristics (Continued)

\begin{tabular}{|c|c|c|c|c|c|c|}
\hline \multirow[t]{2}{*}{ Characteristic } & \multicolumn{2}{|l|}{ Groups } & \multirow[t]{2}{*}{$P$ value } & \multicolumn{2}{|c|}{ Group1 (FTMDT) } & \multirow[t]{2}{*}{$P$ value } \\
\hline & $\overline{1(\text { FTMDT) }}$ & 2(Conventional) & & Subgroup 1a & Subgroup $1 \mathrm{~b}$ & \\
\hline III & $66(38)$ & $69(41)$ & & $36(41)$ & $30(35)$ & \\
\hline IV & $1(1)$ & $0(0)$ & & $1(1)$ & $0(0)$ & \\
\hline Missing & $12(7)$ & $15(8)$ & & $5(6)$ & $7(9)$ & \\
\hline
\end{tabular}

FTMDT Fast-track multi-discipline treatment, $M$ (IQR) Median (interquartile range), F/M Female/male, F\% Percent of females in subgroup, $B M I$ Body mass index, $A S A$ American Society of Anesthesiologists, $n$ number, CEA Carcinoembryonic antigen, CA199 Carbohydrate antigen 19-9, $p T$ pathological T stage, $p N$ pathological $\mathrm{N}$ stage, PTNM pathological 7th edition TNM stage

\section{Quality of life}

The preoperative response rate of questionnaires was higher in group 1 than in group 2 (83.7\% vs. $72.4 \%, P=$ $0.0130)$. The postoperative questionnaire response rates between the two groups were similar at 1 week, 3 months, and 6 months (all $P>0.05$ ) The QLQ-C30 physical functioning scores 1 week after surgery were better in group 1 than in group 2 (80 vs. 66.67, $P=$ 0.0472). The QLQ-C30 fatigue scores 1 week after surgery were also better in group 1 than in group 2 (33.33 vs. $44.44, P=0.0095)$.

\section{Survival}

The median follow-up time was 71 months, with no differences in DFS or OS between the treatment groups (Fig. 2, Table 2). The five-year DFS for groups 1 and 2 were $82.6 \%$ [95\% confidence interval (CI), 75.6-87.8\%] and $80.0 \% \quad(95 \% \mathrm{CI}, 73.0-85.4 \%)$, respectively $(P=$ $0.2780)$. The five-year OS rates of groups 1 and 2 were 87.1\% (95\%CI, 80.7-91.5\%) and $87.1 \%$ (95\%CI $80.8-$ 91.4\%), respectively $(P=0.7420)$ (see in Fig. 2 ).

\section{Discussion}

The concept of FTMDT was the first to integrate medical oncology with a multidiscipline treatment model [13]. This approach means that colorectal cancer is treated as an integrated disease to be followed through a recovery period of 6 months instead of just as a surgical disease. Regarding the primary endpoint, the FTMDT model decreased the total hospital stay. Moreover, the postoperative hospital stay was also shorter in the FTMDT model than in the conventional model. The results are consistent with those of previous studies. ${ }^{8,10-13}$ Within the scope of fast-track surgery procedures, laparoscopic surgery did not reduce the postoperative hospital stay compared to open surgery. The FTMDT trial found that laparoscopic surgery resulted in faster surgical recovery than open surgery did. The morbidity of postoperative in-hospital complications was lower in the FTMDT group than in the conventional treatment group. The five-year DFS and OS were similar between the two groups, which means that the FTMDT model for colorectal cancer is as safe as the conventional treatment model is.
The fast-track surgery protocols used in the FTMDT trial, which were significantly different from the protocols of trials conducted in Western countries, were modified for Chinese patients [13, 18, 19]. Though the concept of fast-track surgery was proposed nearly two decades ago, ${ }^{4,26}$ the practice of the Western model in China was difficult because of the intense doctor-patient relationship and the deep-rooted health-preserving culture in China $[5,6,20]$. Some procedures used in the LAFA and EnROL trials were considered by Chinese surgeons to be radical and possibly dangerous. These included actions on the first day after surgery, such as the oral intake of more than $2 \mathrm{l}$ of liquid, intake of a normal diet, stoppage of intravenous infusion, and getting out bed for more than $6 \mathrm{~h}[18,19]$. Consequently, mandatory changes in the fast-track surgery model were tailored for Chinese patients and surgeons. The current trial verified the safety of the FTMDT model for Chinese patients. The FTMDT model, using modified and moderate fasttrack surgery procedures, reduced the postoperative hospital stay just as the fast-track models in Western trials did. Our results show that fast-track surgery procedures are adaptable to various societies and cultures.

The role of laparoscopic surgery in fast-track protocols is controversial. In contrast, with the LAFA and EnROL trials, we did not find that the laparoscopic operation with FTMDT decreased the postoperative hospital stay compared to the open operation. Additionally, the postoperative hospital stay of 6 days was slightly longer than that in the LAFA/EnROL trials, which was 5 days. This result could be due to differences in the fast-track treatment procedures. Importantly, the open operation performed with the fast-track protocol resulted in shorter postoperative and overall hospital stays than did the laparoscopic operation performed with the conventional treatment. The ongoing trial known as "TAPAS", a prospective cohort study for patients with colon carcinomas, seeks to determine which of three protocols, i.e., traditional open surgery, open fast-track surgery, and laparoscopic fast-track surgery with multimodal management, best minimizes the cost [21]. The open fast-tract surgery generated the lowest surgical costs among all four subgroups in the FTMDT trial. Similar results have also been reported by the LAFA trial. ${ }^{8}$ In that trial, open 


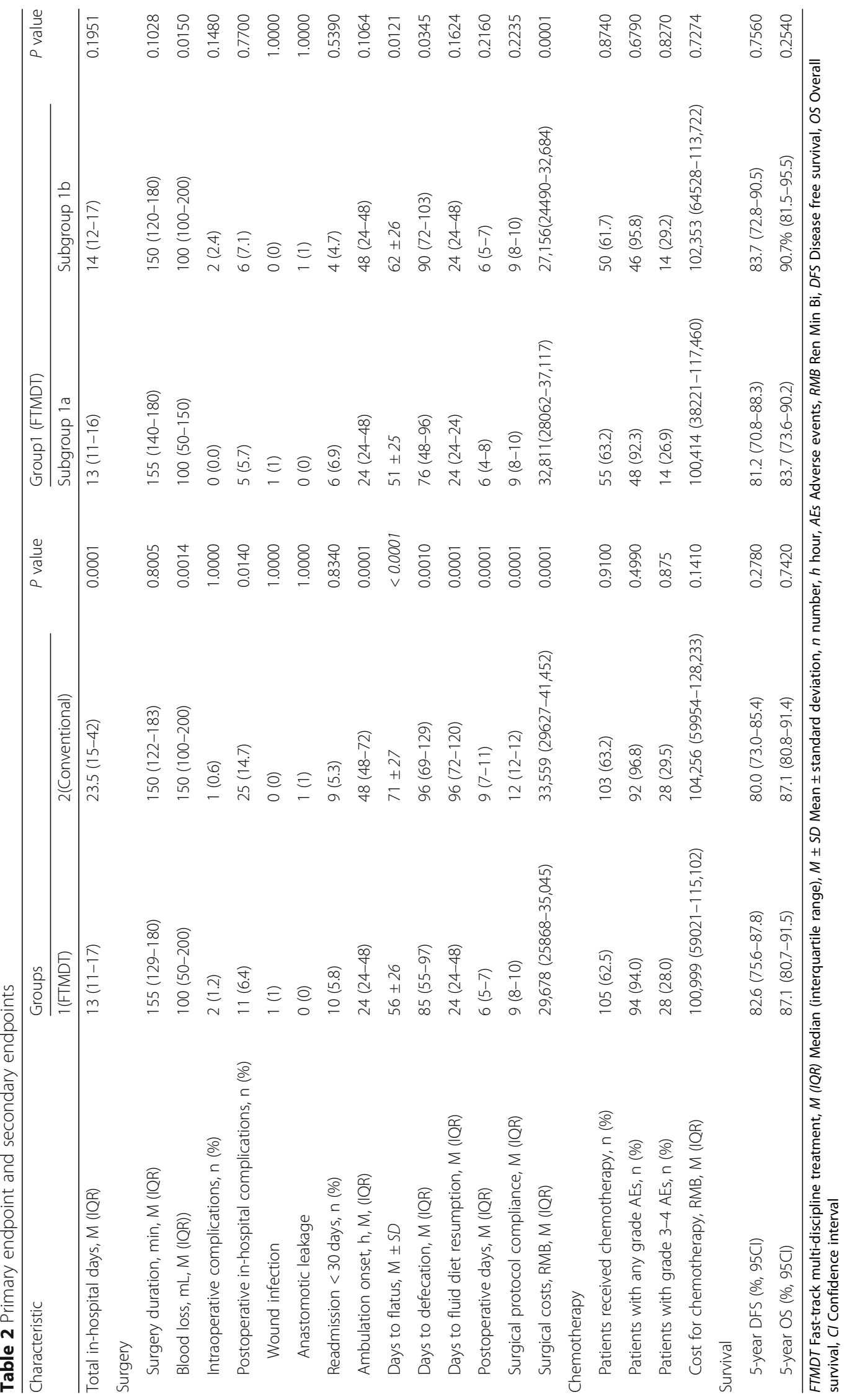




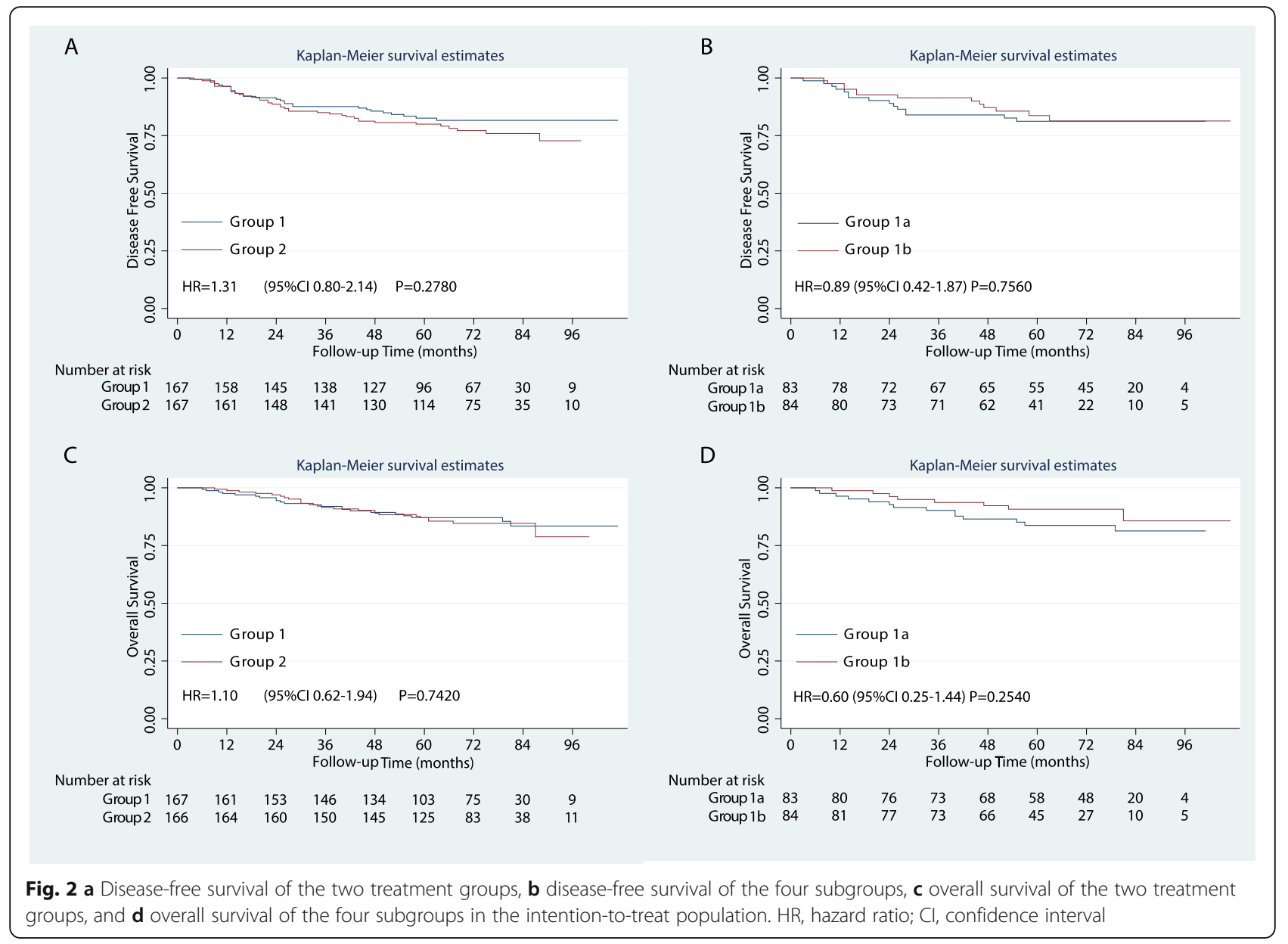

surgery and fast-track procedures achieved a median postoperative hospital stay of 6 days, similar to that achieved with laparoscopy and standard care. In addition, the number of days to attain preoperative levels of solid food tolerance, passage of first flatus, and mobility following open surgery with fast-track procedures was shorter than that with laparoscopy and standard care [8]. Thus, an open operation combined with fast-track treatment is a better choice than laparoscopic operation alone is.

Even though laparoscopic surgery did not significantly reduce the hospital stay more than that required for open surgery in this trial, at least three advantages still back laparoscopy as the best choice for fast-track surgery. First, compared to the open operation, the laparoscopic operation optimized by fast-tract surgical protocols resulted in much less trauma, e.g., less blood loss and reduced time to resumption of flatus and defecation. As a result, laparoscopic surgery decreased the surgical stress and accelerated postoperative nutrition and resumption of immune levels compared to open surgery [22, 23]. Second, laparoscopy ensured that surgeons could proficiently dissect tumors with a high-definition view, thus minimizing the possibility of inadvertent injury. The last but not least advantage of laparoscopy surgery is that it was welcomed by patients. In the EnROL trial, 32\% of potential patients rejected recruitment because they wanted to receive laparoscopy instead of being randomized to the open surgery group [22]. This same concern by patients also slowed recruitment for the FTMDT trial in the first 2 years.

There were several limitations in this trial. First, the fast-track surgery procedures were more conservative than the Western procedures are. The median number of checkpoints that met compliance for fast-track treatment in this trial was 9 of 13 surgical checkpoints; thus only $69.2 \%$ of the fast-track procedures were complied with by the patients and surgeons. Second, the FTMDT perioperative treatment was affected by new and better understandings of perioperative procedures that were acquired during the trial itself. For instance, both groups of patients should have received bowel preparation as required by the protocol; however, only $64.3 \%$ patients in the FTMDT group received it. Third, the recruited patients in this trial were younger than the patients in the Western trials. The median age of colorectal cancer onset in China is approximately 10 years earlier than it is in Western countries [3]. The ASA scores, BMI, and 
morbidity of postoperative complications were lower in our trial than in the Western trials. Fourth, this trial was not conducted using a blinded protocol which may have contributed to intervention bias. For statistics, only simple randomization was adopted without stratification. The study involved both surgery and adjuvant chemotherapy, with four subgroups making the design complex and potentially imbalanced. Considering the type I error wasn't adjusted by 2 groups, the sample size may not be enough to explain the secondary end points as there were also many confounding factors.

While the fast-track treatment with open surgery had some economic advantages, the laparoscopic surgery had minor advantages over open surgery for postoperative recovery. The integration of laparoscopic surgery, fasttrack treatment, and XELOX chemotherapy in FTMDT represents an optimal model to enhance patient recovery from surgical resection of colorectal cancer.

\section{Conclusions}

The FTMDT model, which integrates laparoscopic surgery, fast-track treatment, and XELOX chemotherapy, was the superior model for enhancing the recovery of Chinese patients with colorectal cancer.

\begin{abstract}
Abbreviations
ASA: American Society of Anesthesiologists; BMI: Body mass index; Cl: Confidence interval; CRFs: Case report forms; DFS: Disease-free survival; EORTC: European Organization for Research and Treatment; FOLFOX: Adjuvant chemotherapy with leucovorin, fluorouracil, and oxaliplatin; FTMDT: Fast-track multidisciplinary treatment; IQR: Interquartile ranges; NCI CTCAE: National Cancer Institute Common Terminology Criteria for Adverse Events; OS: Overall survival; XELOX: Adjuvant chemotherapy with capecitabine and oxaliplatin
\end{abstract}

\section{Acknowledgements}

The authors have declared no conflicts of interest. We gratefully thank Britt Bromberg, PhD, ELS, of Xenofile Editing (www.xenofileediting.com) for editing assistance with the manuscript.

\section{Authors' contributions}

$J \mathrm{~L}$ conceptualized this study and designed the methods. He also collected data at the Second Affiliated Hospital of Zhejiang University. He analyzed the final data and wrote the manuscript. XXK helped to design the statistical methods. He collected data at the Second Affiliated Hospital of Zhejiang University. He also analyzed the final data and helped write the manuscript. JJZ helped to design the methods. She collected data at the Second Affiliated Hospital of Zhejiang University. She also analyzed the final data and helped revise the manuscript. YMS helped to design the methods. He helped perform the operations and collected data at the Second Affiliated Hospital of Zhejiang University. He also analyzed the final data and helped revise the manuscript. XFH and MC helped to design the methods. They helped perform the operations and collected data at the Sir Run Shao Hospital. They also analyzed they final data and helped revise the manuscript. GHL helped to design the methods. He helped perform the operations and collected data at People's Hospital of Yuyao. He also analyzed the final data and helped revise the manuscript. XJY helped to design the methods. He helped perform the operations and collected data at People's Hospital of Shaoxing. He also analyzed the final data and helped revise the manuscript. XYD helped to design the methods. He helped perform the operations and collected data at Ningbo No. 2 Hospital. He also analyzed the final data and helped revise the manuscript. ML helped to design the methods. He helped perform the operations and collected data at the Second Affiliated Hospital of Wenzhou Medicine College. He also analyzed the final data and helped revise the manuscript. KJ, DLF, XLL, JJH, JWW, LFS, DX helped to design the methods. They helped perform the operations and collected data at the Second Affiliated Hospital of Zhejiang University. They also analyzed the final data and helped revise the manuscript. JYX and MY helped to design the anesthesia methods. They helped perform anesthesia during the operations and collected data at the Second Affiliated Hospital of Zhejiang University. They also analyzed the final data and helped revise the manuscript. YT and JSL helped to design the statistical methods. They summarized the data from all centers and confirmed the statistical results. They also helped revise the manuscript. YY helped to design the chemotherapy strategies. She helped perform anesthesia during the operations and collected data at the Second Affiliated Hospital of Zhejiang University. She also helped revise the manuscript. KFD conceptualized this study and designed the trial. He also collected data at the Second Affiliated Hospital of Zhejiang University. He analyzed the final data and revised the manuscript. We declare that all the authors listed above have read and approved of the final version of this manuscript.

\section{Funding}

The study was supported by National Key R\&D Program of China (2017YFC0908200), the National Natural Science Foundation of China (81672916; 81301890), and the Key Technology Research and Development Program of Zhejiang Province (No. 2017C03017). The sponsors played no role in the study design, data collection or interpretation, or analysis, or decision to submit the article for publication.

\section{Availability of data and materials}

The present article is a RCT research, and the data contained identifying/ confidential patient data so it is no available.

Ethics approval and consent to participate

This trial was approved by the Ethics Committee of Second Affiliated Hospital Zhejiang University School of Medicine (2010LSY No. 6). All patients were screened by the investigators and signed informed consents.

\section{Consent for publication}

Not Applicable.

\section{Competing interests}

The authors declare that they have no competing interests.

\section{Author details}

${ }^{1}$ Department of Colorectal Surgery and Cancer Institute (Key Laboratory of Cancer Prevention and Intervention, China National Ministry of Education; Key Laboratory of Molecular Biology in Medical Sciences, Zhejiang Province, China), the Second Affiliated Hospital of Zhejiang University School of Medicine, Hangzhou, China. ${ }^{2}$ Department of Anus and Large Intestine, Sir Run Shaw Hospital, Zhejiang University College of Medicine, No. 3 East Qingchun Road, Hangzhou 310016, Zhejiang Province, China. ${ }^{3}$ Department of Anus and Large Intestine, People's Hospital of Yuyao, 800 City Road East, Yuyao 315400, Zhejiang Province, China. ${ }^{4}$ Department of Anorectum, People's Hospital of Shaoxing, 568 Zhong-Xing North Rd, Shaoxing 312000, Zhejiang Province, China. ${ }^{5}$ Department of Anus and Large Intestine, Ningbo No. 2 Hospital, No. 41 Northwest Road, Ningbo 315010, Zhejiang Province, China. ${ }^{6}$ Department of Anus and Large Intestine, Second Affiliated Hospital, Wenzhou Medicine College, 109 Xue-Yuan West Rd, Wenzhou 325027, Zhejiang Province, China. ${ }^{7}$ Department of Anesthesiology, Second Affiliated Hospital, Zhejiang University School of Medicine, No. 88 Jiefang Road, Hangzhou 310009, Zhejiang Province, China. ${ }^{8}$ Engineering Research Center of EMR and Intelligent Expert System, Ministry of Education, Collaborative Innovation Center for Diagnosis and Treatment of Infectious Diseases, College of Biomedical Engineering and Instrument Science, Zhejiang University, No. 38 Zheda Road, Hangzhou 310027, Zhejiang, China. ${ }^{9}$ Department of Medical Oncology, Second Affiliated Hospital, and The Key Laboratory of Cancer Prevention and Intervention, China National Ministry of Education, Zhejiang University School of Medicine, No. 88 Jiefang Road, Hangzhou 310009, Zhejiang Province, China. 
Received: 2 December 2018 Accepted: 23 September 2019

Published online: 23 October 2019

\section{References}

1. Siegel RL, Miller KD, Fedewa SA, Ahnen DJ, Meester RGS, Barzi A, Jemal A. Colorectal cancer statistics, 2017. CA Cancer J Clin. 2017;67(3):177-93.

2. Siegel RL, Miller KD, Jemal A. Cancer statistics, 2017. CA Cancer J Clin. 2017; 67(1):7-30.

3. Chen W, Zheng R, Baade PD, Zhang S, Zeng H, Bray F, Jemal A, Yu XQ, He J. Cancer statistics in China, 2015. CA Cancer J Clin. 2016;66(2):115-32.

4. Kehlet $\mathrm{H}$, Wilmore DW. Evidence-based surgical care and the evolution of fast-track surgery. Ann Surg. 2008;248(2):189-98.

5. Gustafsson UO, Scott MJ, Schwenk W, Demartines N, Roulin D, Francis N, McNaught CE, Macfie J, Liberman AS, Soop M, et al. Guidelines for perioperative care in elective colonic surgery: enhanced recovery after surgery (ERAS((R))) society recommendations. World J Surg. 2013;37(2):259-84.

6. Nygren J, Thacker J, Carli F, Fearon KC, Norderval S, Lobo DN, Ljungqvist O, Soop M, Ramirez J. Guidelines for perioperative care in elective rectal/pelvic surgery: enhanced recovery after surgery (ERAS((R))) society recommendations. World J Surg. 2013;37(2):285-305.

7. Kennedy RH, Francis EA, Wharton R, Blazeby JM, Quirke P, West NP, Dutton SJ. Multicenter randomized controlled trial of conventional versus laparoscopic surgery for colorectal cancer within an enhanced recovery programme: EnROL. J Clin Oncol. 2014;32(17):1804-11.

8. Vlug MS, Wind J, Hollmann MW, Ubbink DT, Cense HA, Engel AF, Gerhards MF, van Wagensveld BA, van der Zaag ES, van Geloven AA, et al. Laparoscopy in combination with fast track multimodal management is the best perioperative strategy in patients undergoing colonic surgery: a randomized clinical trial (LAFA-study). Ann Surg. 2011;254(6):868-75.

9. Feng F, Li XH, Shi H, Wu GS, Zhang HW, Liu XN, Zhao QC. Fast-track surgery combined with laparoscopy could improve postoperative recovery of lowrisk rectal cancer patients: a randomized controlled clinical trial. J Dig Dis. 2014;15(6):306-13.

10. Ren L, Zhu D, Wei Y, Pan X, Liang L, Xu J, Zhong Y, Xue Z, Jin L, Zhan S, et al. Enhanced recovery after surgery (ERAS) program attenuates stress and accelerates recovery in patients after radical resection for colorectal cancer: a prospective randomized controlled trial. World J Surg. 2012;36(2):407-14.

11. Wang G, Jiang ZW, Zhao K, Gao Y, Liu FT, Pan HF, Li JS. Fast track rehabilitation programme enhances functional recovery after laparoscopic colonic resection. Hepatogastroenterology. 2012;59(119):2158-63.

12. Yang $D, H e W$, Zhang $S$, Chen $H$, Zhang $C$, He Y. Fast-track surgery improves postoperative clinical recovery and immunity after elective surgery for colorectal carcinoma: randomized controlled clinical trial. World J Surg. 2012;36(8):1874-80.

13. Zhou JJ, Li J, Ying XJ, Song YM, Chen R, Chen G, Yan M, Ding KF. Fast track multi-discipline treatment (FTMDT trial) versus conventional treatment in colorectal cancer--the design of a prospective randomized controlled study. BMC Cancer. 2011:11:494.

14. Otchy D, Hyman NH, Simmang C, Anthony T, Buie WD, Cataldo P, Church J, Cohen J, Dentsman F, Ellis CN, et al. Practice parameters for colon cancer. Dis Colon Rectum. 2004;47(8):1269-84.

15. Ding KF, Chen R, Zhang JL, Li J, Xu YQ, Lv L, Wang XC, Sun LF, Wang JW, Zheng $S$, et al. Laparoscopic surgery for the curative treatment of rectal cancer: results of a Chinese three-center case-control study. Surg Endosc. 2009;23(4):854-61.

16. Li M, Gu J. Changing patterns of colorectal cancer in China over a period of 20 years. World J Gastroenterol. 2005;11(30):4685-8.

17. Li J, Chen R, Xu YQ, Wang XC, Zheng S, Zhang SZ, Ding KF. Impact of a laparoscopic resection on the quality of life in rectal cancer patients: results of 135 patients. Surg Today. 2010;40(10):917-22.

18. Wind J, Hofland J, Preckel B, Hollmann MW, Bossuyt PM, Gouma DJ, van Berge Henegouwen Ml, Fuhring JW, Dejong CH, van Dam RM, et al. Perioperative strategy in colonic surgery; LAparoscopy and/or FAst track multimodal management versus standard care (LAFA trial). BMC Surg. 2006;6:16.

19. Kennedy RH, Francis A, Dutton S, Love S, Pearson S, Blazeby JM, Quirke P, Franks PJ, Kerr DJ. EnROL: a multicentre randomised trial of conventional versus laparoscopic surgery for colorectal cancer within an enhanced recovery programme. BMC Cancer. 2012;12:181.

20. Yang T, Zhang H, Shen F, Li JW, Wu MC. Appeal from Chinese doctors to end violence. Lancet (London, England). 2013;382(9906):1703-4.
21. Reurings JC, Spanjersberg WR, Oostvogel HJ, Buskens E, Maring J, Kruijt F, Rosman C, van Duivendijk P, Dejong CH, van Laarhoven CJ. A prospective cohort study to investigate cost-minimisation, of traditional open, open fAst track recovery and laParoscopic fASt track multimodal management, for surgical patients with colon carcinomas (TAPAS study). BMC Surg. 2010;10:18.

22. Veenhof $A A$, Vlug MS, van der Pas MH, Sietses $C$, van der Peet $D L$, de Lange-de Klerk ES, Bonjer HJ, Bemelman WA, Cuesta MA. Surgical stress response and postoperative immune function after laparoscopy or open surgery with fast track or standard perioperative care: a randomized trial. Ann Surg. 2012;255(2):216-21.

23. Xu D, Li J, Song Y, Zhou J, Sun F, Wang J, Duan Y, Hu Y, Liu Y, Wang X, et al. Laparoscopic surgery contributes more to nutritional and immunologic recovery than fast-track care in colorectal cancer. World J Surg Oncol. 2015;13:18

\section{Publisher's Note}

Springer Nature remains neutral with regard to jurisdictional claims in published maps and institutional affiliations.
Ready to submit your research? Choose BMC and benefit from:

- fast, convenient online submission

- thorough peer review by experienced researchers in your field

- rapid publication on acceptance

- support for research data, including large and complex data types

- gold Open Access which fosters wider collaboration and increased citations

- maximum visibility for your research: over $100 \mathrm{M}$ website views per year

At BMC, research is always in progress.

Learn more biomedcentral.com/submissions 